\title{
Performance of undoped BGO crystals under extremely high dose conditions
}

\author{
K.C. Peng ${ }^{\mathrm{a}}$, M.Z. Wang ${ }^{\mathrm{a}, *}$, C.H. Wang ${ }^{\mathrm{b}}$, K. Ueno ${ }^{\mathrm{a}}$, H.C. Huanga, Y.H. Chang ${ }^{\mathrm{c}}$, \\ F.I. Chou ${ }^{\mathrm{d}}$, W.S. Hou ${ }^{\mathrm{a}}$, Y.Y. Weid \\ ${ }^{a}$ Department of physics, National Taiwan University, Taipei, Taiwan \\ ${ }^{\mathrm{b}}$ National Lien Ho College of Tech. and Commerce, Miao Li, Taiwan \\ ${ }^{c}$ National Central University, Chung Li, Taiwan \\ ${ }^{\mathrm{d}}$ NSTDC of National Tsing Hua University, Hsin-Chu, Taiwan
}

Received 6 October 1998

\begin{abstract}
We checked the performance of undoped BGO crystals under extremely high dose conditions (dosage up to 88 Mrad). The light outputs of the crystals drop by $\sim 30 \%$ after receiving 10 Mrad of $\gamma$-ray dose but remain stable throughout the rest of the test. The self-recovery behavior of BGO under $20 \mathrm{Mrad}$ has also been studied. The reduced variation in light output from pre-radiated BGOs, together with a fixed recovery limit, indicate that a pre-radiation process may be helpful to stabilize the BGO performance in a low dose environment. (C) 1999 Elsevier Science B.V. All rights reserved.
\end{abstract}

Keywords: BGO; Dose; Recovery

\section{Introduction}

Following the material study of Ref. [1], we impose higher dose on undoped bismuth germanate $\left(\mathrm{Bi}_{4} \mathrm{Ge}_{3} \mathrm{O}_{12}\right)$ crystals, which has been known as BGO and extensively used for electromagnetic calorimetry in high-energy physics experiments [2-4]. The advantages of BGO are its excellent $e / \gamma$ energy resolution $(0.3-1 \% / \sqrt{E(\mathrm{GeV})})$, high density,

* Corresponding author.: Fax: + 886-2-23693472.

E-mail address: mwang@phys.ntu.edu.tw (M.Z. Wang) short radiation length, large refractive index, suitable scintillating properties (fast decay time of $\sim 300 \mathrm{~ns}$ and peak scintillation at $\sim 450 \mathrm{~nm}$ ) and non-hygroscopic nature. Our group is constructing such a calorimeter to improve coverage of small angles around the beam pipe of the BELLE detector at KEK B-factory. Due to the proximity of such calorimeters to the electron/positron beam pipes and the interaction point, radiation level is rather high for intense flux of synchrotron radiation, beam-beam interactions, and beam-gas background events. Therefore, it is necessary for us to study the radiation hardness and recovery mechanism of BGO crystals. 


\section{Experimental setup}

The schematic diagram of the setup in the irradiation facility of National Tsing Hua University, Taiwan, is shown in Fig. 1. The radioactive source is ${ }^{60} \mathrm{Co}$ with cylindrical shape and with radioactivity of $\sim 1000 \mathrm{C}$. The diameter of the ${ }^{60} \mathrm{Co}$ cast is $3 \mathrm{~cm}$, and the holder diameter is $6 \mathrm{~cm}$.

Immediately after each irradiation, the samples were taken out from the irradiation cell to a nearby experimental booth. The trapezoid BGOs have been separated into two sets: LBGO (longitudinally irradiated) and TBGO (transversely irradiated). We define the equivalent dose as the amount of dose

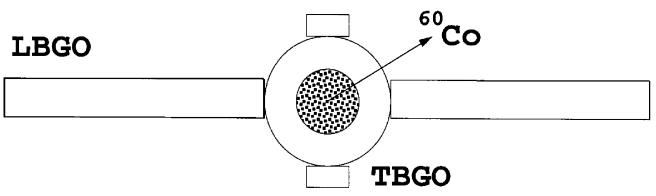

Fig. 1. Top view of experimental setup in radiation source room. BGOs were $3 \mathrm{~cm}$ away from the centre of $\sim 1000 \mathrm{C}{ }^{60} \mathrm{Co}$. that would have been absorbed by water if placed at the position of the front surface of the sample. The average dose absorbed by the TBGO is about the same as the equivalent dose, where the average dose absorbed by the LBGO is about $40 \%$ of the equivalent dose [1].

Total of 10 pieces of undoped trapezoid BGO crystals with approximate size of $12 \times 2 \times 1 \mathrm{~cm}^{3}$, produced at the Institute of Inorganic Chemistry, Novosibirsk, Russia, were used in this irradiation test. Two out of ten BGOs were used as reference crystals. The rest were separated into two sets: four BGOs (two LBGOs and two TBGOs) were used for high dose study and the other four TBGOs were later monitored for their self-recovery.

We measured the light output of all BGOs with $90 \mu \mathrm{C}$ radioactive source ${ }^{137} \mathrm{Cs}$. A lead collimator focuses $\gamma \mathrm{s}$ within $2 \mathrm{~mm}$ hole and $15 \mathrm{~mm}$ depth onto BGOs. A photomultiplier tube (PMT), Hamamatsu R329-05, 2-in. in diameter, was used for reading out the light output from test samples. A reference crystal (labeled as $\mathrm{BGO}_{\mathrm{r}}$ ) was in the


Fig. 2. Results of the first week irradiation. The horizontal scale is in equivalent dose and the vertical scale is a ratio between BGO and $\mathrm{BGO}_{\mathrm{r}}$, with first point normalized to 1 (a) and (b) were irradiated longitudinally; (c) and (d) suffered more radiation damage for being irradiated transversely. 
cycle of testings in front of PMT to simultaneously cancel out any variation due to the changes in the environment.

The signals from the PMT were gated by a selftrigger and digitized with a LeCroy CAMAC ADC 2249A. The CAMAC crate was controlled by a PC-based data acquisition (DAQ) system. The gate width was set to be 800 ns for complete integration. with a pulser, we made empty signal triggers and accumulated pedestal events simultaneously.

\section{Results}

We had two week-long experiments. In the first week, we concentrated on studying the performance under high dose irradiation. It was a con- tinuous operation and the crystals were subjected to irradiation most of the time. The results are shown in Fig. 2. It is clear that all crystals show a stable performance after the first point. We observe no change up to $88 \mathrm{Mrad}$. However, the decrease in light output is quite dependent on the individual crystal. On average, the drop is about $30 \%$. The performance characteristics are about the same for both LBGOs and TBGOs.

In the second week, we were allowed having access to a new facility with a 30 times stronger ${ }^{60}$ Co source, but without our DAQ system nearby. We tested 5 new identical BGOs, with one as reference. Two of them were irradiated to $10 \mathrm{Mrad}$ and two to $5 \mathrm{Mrad}$, all in $5 \mathrm{~h}$ and all transversely. The light output decreased by $\sim 30 \%$. We found a slow recovery after 3 days which recovered to $\sim 90 \%$ of

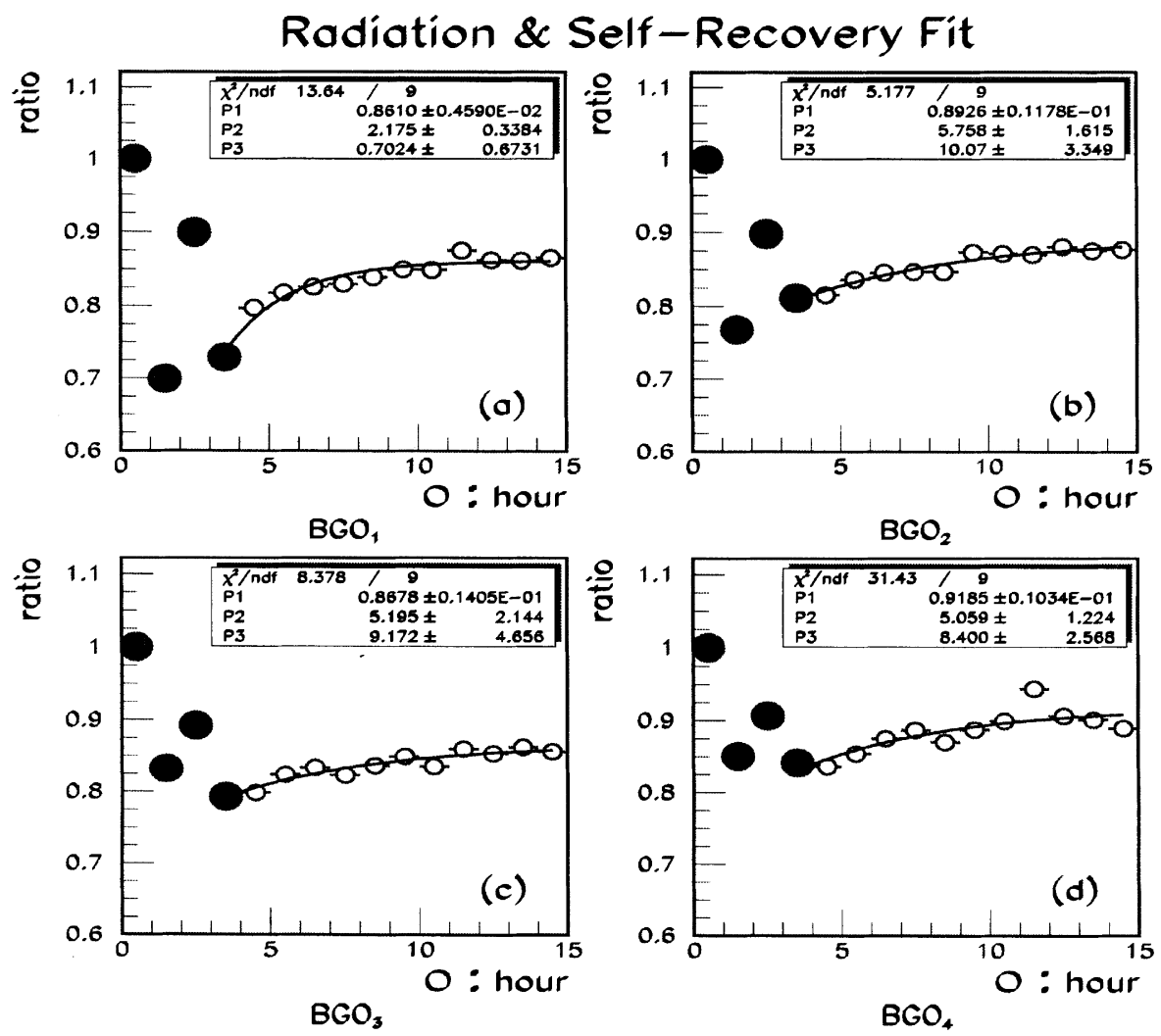

Fig. 3. Results of the second week irradiation and self-recovery monitoring. The horizontal scale is in hour for open circle data points, and the vertical scale is a ratio of different samples to $\mathrm{BGO}_{\mathrm{r}}$, with first point normalized to 1 . The dark points are not scaled horizontally. See text for more information. The fits through open circles show self-recovery time constants at room temperature after the second irradiation of $10 \mathrm{Mrad}$ in (a),(b) and $5 \mathrm{Mrad}$ in (c),(d). The statistical error on each data point is less than $1 \%$. 
their original light output. Then, we sent these 3-day-recovered BGOs back for another round of irradiation with the same amount of dosage. Hourly measurements were performed immediately after the irradiation to monitor the BGO self-recovery. The results are shown in Fig. 3.

Unlike Ref. [5], we only assume one exponential time constant for this recovery, although there may be other time constants that form multiple exponentials to portray very fast recovery (less than an hour), week-long self-recovery, etc. The fitting function is chosen to be

$P_{1} \times\left(1-\mathrm{e}^{-\left(t+P_{3}\right) / P_{2}}\right)$.

$P_{1}$ represents the dimensionless upper limit for self-recovery of particular BGO in an unlimited time. $P_{3}$ is just a constant of shifting data points. Finally, $P_{2}$ is the self-recovery time constant.

Some caution must be taken for the interpretation of the first four data points since they are not scaled horizontally. The interval between points 1 and 2 is about $8 \mathrm{~h}$ including $5 \mathrm{~h}$ of irradiation and $3 \mathrm{~h}$ of returning to our Lab. The interval of points $2-3$ is a 72-h self-recovery. The interval between points 3 and 4 is $6 \mathrm{~h}$ with $5 \mathrm{~h}$ of irradiation and $1 \mathrm{~h}$ of transit. From point 4 on, the intervals are in units of hours and fits are also shown. Due to self-recovery during transportation, the relative light output after irradiation should be smaller than the values shown in points 2 and 4 . Note that the relative drop is considerably reduced for the second irradiation of the same amount of dose. Another report is now in preparation for a better determination of the recovery time constant and a comparison with low dose data [6].

\section{Conclusion}

In this study, we observe a stable performance of undoped BGO crystals under very high dose conditions. However, the light output decrease by $\sim 30 \%$ after receiving $\sim 10$ Mrad dose. The crystals may have suffered some permanent damage but can recover to $90 \%$ of their original light output in a period of a day. The reduced variation in light output and the same upper limit of recovery for pre-radiated samples, shown in Fig. 3, indicate that it is a good idea to pre-irradiate all of the crystals before installing them into BELLE.

\section{Acknowledgements}

We would like to thank the staffs of the irradiation facilities at NTHU for their hard work and kind assistance. This experiment is supported by the grant NSC 86-2112-M-002-026 of the Republic of China.

\section{References}

[1] S.K. Sahu et al., Nucl. Instr. and Meth. A 388 (1997) 144.

[2] L3 Collaboration, R. Summer et al., Nucl. Instr. and Meth. A 265 (1988) 252.

[3] H. Hayashii et al., Nucl. Instr. and Meth. A 316 (1992) 202.

[4] D.N. Grigoriev et al., IEEE Trans. Nucl. Sci. 42 (1995) 505.

[5] P. Lecoq et al., Nucl. Instr. and Meth. A 300 (1991) 240.

[6] K.C. Peng et al., Belle Note 205, 1997. 